\title{
Hardware Design of Electro-Mechanical Actuator System
}

\author{
Cheng $\mathrm{Wu}^{1}$, Huailin $\mathrm{Zhao}^{1}$, Jihong $\mathrm{Zhu}^{2}$
}

\author{
${ }^{1}$ School of Electrical and Electronic Engineering, Shanghai Institute of Technology, Shanghai, China \\ ${ }^{2}$ Department of Computer Science \& Technology, Tsinghua University, Beijing, China
}

Keywords: electromechanical actuator, dynamic response, static performance, LVDT

\begin{abstract}
As the important executive body of flight control system, the servo aims to drive the control surface deflection according to the position command. This paper makes a hardware design for a digital electric-mechanical servo controller for valve adjustment. Three-phase brushless DC motor(BLDCM) acts as the core executive body. The system selects DSP + CPLD as the main control unit and the high-precision Linear Variable Differential Transformer (LVDT) as the position sensor. We achieve the digital control through the realization of three closed-loop control strategy including current loop, speed loop and position loop. Experimental results show that the system has high dynamic response speed and great precision static performance.
\end{abstract}

\section{Introduction}

The actuator is a high precision and response position servo system, which manipulates control surface deflection to change the flight attitude or trajectory of missiles according to the received position command from the flight control system ${ }^{[1-3]}$. The common servos can be divided into pneumatic servos, hydraulic servos and electric servos by the power source. Compared to pneumatic and hydraulic servos, electric servos have such advantages of optimized onboard energy management, easy maintenance and strong battlefield survival, thusto be more widelyused inthe flight controlsystem. ${ }^{[4-5]}$ Electric servos can be divided analog servos and digital servos according to the difference of control mode. Compared withtraditional analogservos, the digital servo control system with the core of high-speed microcontroller had a highdegree of standardization of hardwarecircuit and low productioncost, and greatlyenhancedtheperformanceand reliability ofthe servocontroller ${ }^{[6]}$.

\section{System architecture design}

The servo system is mainly composedof four partsincluding three-phasebrushless DC motor (BLDCM), drive controller, sensors and mechanical structures. The drive controlleristhe most criticalpart in the system design. The overall system block diagram is shown in Fig.1.

As shown, the system accepts master a given angular position command, which acts as the input of position closed-loop controller through the second-order low-pass filter to filter out high frequency noise, at the same time becomes as the input of speed closed-loop controller after processed by the feedforward controller. Feedforward controlisdifferentiatingposition command to improve thesystem dynamicresponse effectively.

The actual angle of the rocker is measured by a position sensor and filtered out high frequency noiseby a first-orderinertialfilter. The filtered actual position and the filtered position command act as the inputs of the position closed-loop. The output of the position loop serves as given quantity of the speed, the actual speed and the output of the feedforward controller act as the input of the speed closed-loop controller together. The speed closed-loop exports the given value of the motor current. The command current combines the actual current which is measured by the current sensor to constitute the currentloop controller. The current loop would export pulse width modulation (PWM) wave, which associates with the Hall signal to gain the conduction state of power MOSFETs through the decoding logic to control the motor rotation. The output shaft of the motor connects to the precision reducer and conducts mechanicaltransformation to drive the servo armswing. 


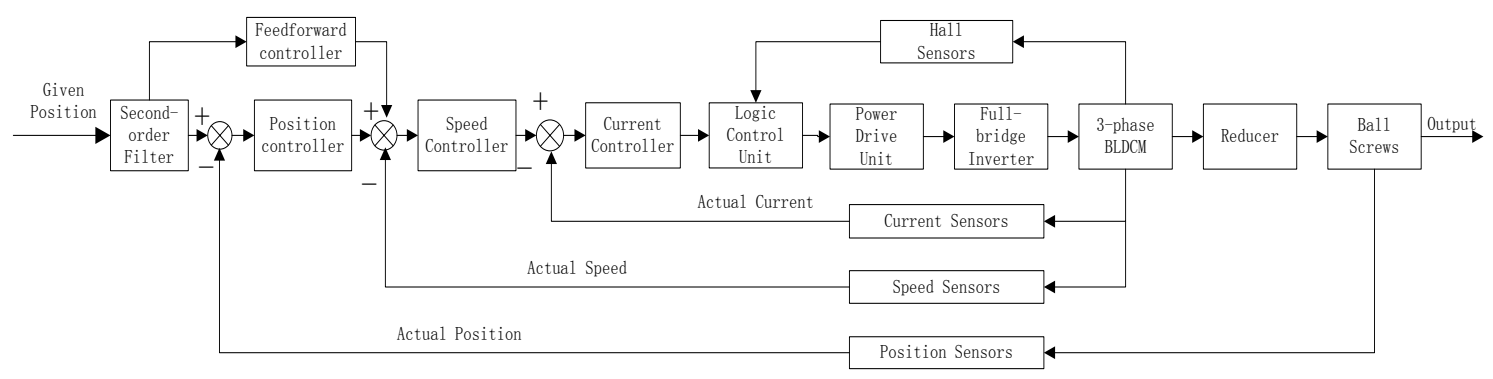

Fig.1 Overallblock diagram of thesystem

\section{Design for the DSP + CPLDdigital control unit}

DSP and CPLD are the main component parts for the digital control unit of the system. DSP has powerful computing capabilities and rich peripheral resources to reduce the number of system components and to save the cost. The system intends to use TMS320F2808 which belongs to the TI as the master controller. CPLD selects the product of the Lattice and the programming is convenient and rich in resources. The block diagram ofthe controller is shown in Fig.2.

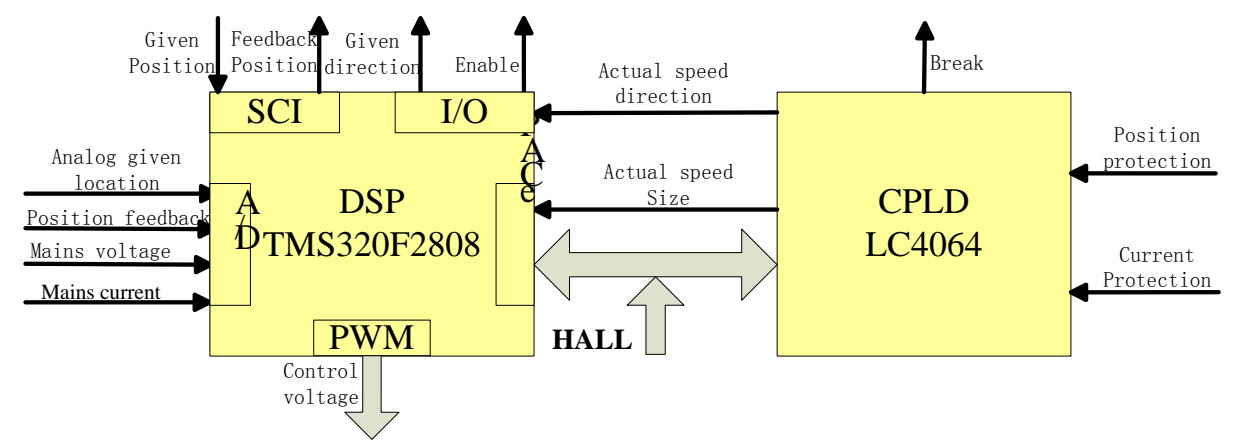

Fig.2 Main control unit

DSPis mainlyresponsible for achievingclosed-loopcontrol and dataprocessingoperations of the system, while the richperipheralresources completeother requirementsfunction. The ePWM module is designed for the purpose of exporting the closed-loop results. The A/D transformation unit is used for the analog acquisition. The SCI module achieves the data communication with the master device by the RS-422 protocol. The eCAP is used to capture the processed Hall sequence to gain the size of the motor speed. The CPLD conducts the Hall pulse triple frequency, simultaneouslycalculatesthe actual motorrotation direction. The design of the position and current protection logic will be finished in CPLD according to the actual needs.

\section{Three-phase full-bridge and drive unit}

The system selectsL6235 which is highly integratedthree-phasebrushless DC motordriver chipas the coreof the drive unit. The device includes all the circuitry needed to drive a three-phase BLDC motor including: a three-phase DMOS bridge, a constant off time PWM current controller and decoding logic for single ended hall sensors that generates the required sequence for the power stage. Besides, it features a non-dissipative overcurrent protection on the high side power MOSFETs and thermal shutdown. The structure of the full-bridge and drive unit is shown Fig. 3. 


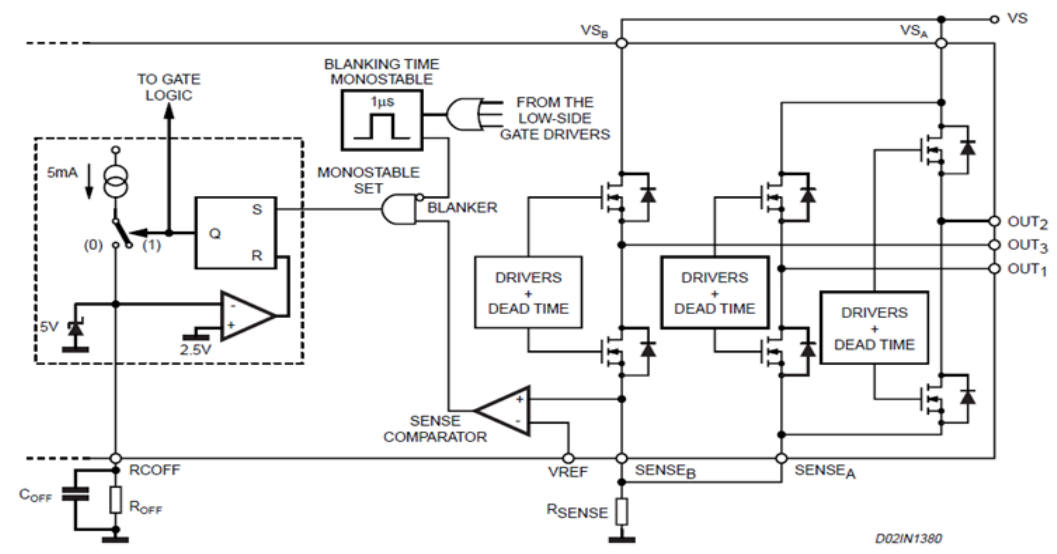

Fig.3 Full-bridge and drive unit block diagram

The motor current controller is built for current closed-loop control in the chip. As shown in figure 3, it has a low bias fast comparator, whose inputs are connected to VREF and SENSE pins. The current control circuit senses the bridge current by sensing the voltage drop across an extern sense resistor connected between the source of the three lower power MOS transistors and ground. As the current in the motor increases the voltage across the sense resistor increases proportionally. The VREF is provided by the output of the speed closed-loop for the purpose of the given of the current closed-loop control. When the voltage drop across the sense resistor becomes greater than the voltage at the reference input pin VREF, the sense comparator triggers the monostable switching the bridge off. When the monostable times out, the bridge will again turn on. The appropriate choice of the external RC network that connected to the RCOFF pin can determines the off-time. The voltage drop across the motor current waveform IOUT and the voltage drop across the current sensing resistor VSENSE are shown in Fig.4.

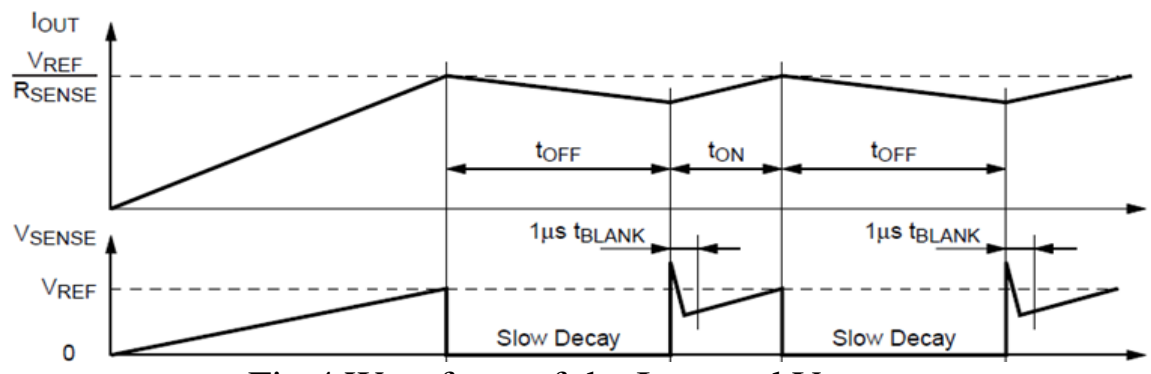

Fig.4 Waveform of the $\mathrm{I}_{\mathrm{OUT}}$ and $\mathrm{V}_{\mathrm{SENSE}}$

\section{The signal acquisition and conditioning module}

The actuator is a position servo system to track the command of the arm angle. So the premise must be guaranteed to gain the accurate actual position. The output shaft of the motor connects to the ball screw after decelerating in order to transform the high-speed rotation of the motor into thrust linear output. At last the ball screw will push the arm swing of the actuator to gain the high torque. The linear variable differential transformer (LVDT) is connected with the ball screw to measure the screw displacement which is converted to the angle of the actuator arm by quantitative relationship.

The internal of the LVDT adopts non-contact measurement to improve the work life while ensuring the accuracy. When the sensor is coupled with the power supply of $12 \mathrm{~V}$, the regulated DC voltage output will vary from $0.5 \mathrm{~V}$ to $4.5 \mathrm{~V}$ according to the displacement. The result will be converted to the range of $0 \sim 3 \mathrm{~V}$ for the A/D module of the DSP by the signal conditioning circuitry. Due to the limited range of the screw displacement, the protected function of the positon need to be designed in order to ensure the safe and stable operation. The output conditioning circuit of the position is shown in Fig.6. 

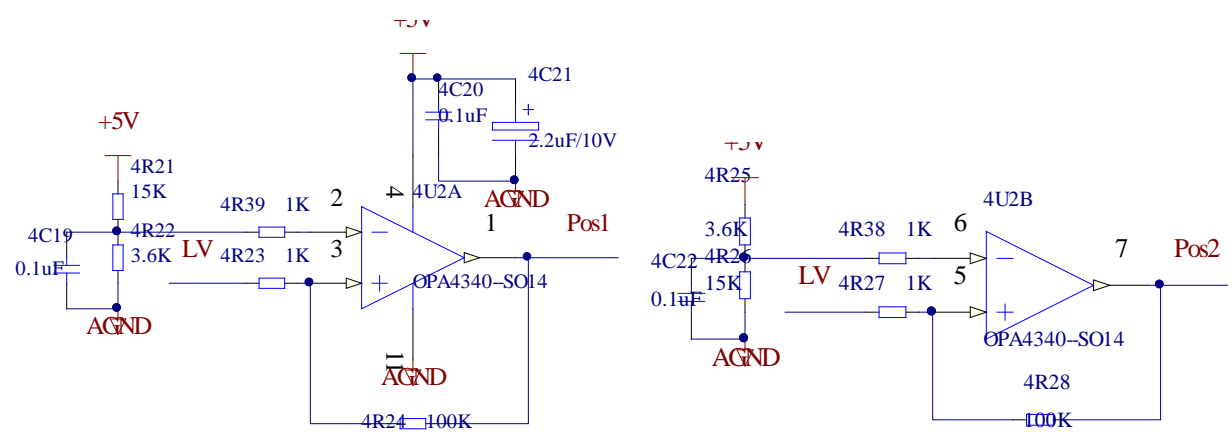

Fig. 5 The conditioning circuit of the position

The conditioning signals Pos1 and Pos2 will be sent to the CPLD for the logic operations. And the system controls normal or break of the motor to achieve the position protection by the driven module according to the results of the above. The logic truth table of the position protection is shown in Table 1.

The position protection module allows the servo arm to rotate properly in the protected areas. The DIR is the given signal of the rotation direction. When the rocker is outside protected areas and the system receives the command rotation direction to the protected areas, the motor rotates normally. Otherwise, when the system receives the opposite direction, all of the MOSFETs of the driven chip will be off and the motor break immediately.

\begin{tabular}{|l|r|r|r|r|r|c|}
\hline POS1 & 1 & 1 & 1 & 1 & 0 & 0 \\
\hline POS2 & 1 & 1 & 0 & 0 & 0 & 0 \\
\hline DIR & 1 & 0 & 1 & 0 & 1 & 0 \\
\hline BRK & 0 & 1 & 1 & 1 & 1 & 0 \\
\hline STATUS & break & reverse & forward & reverse & forward & break \\
\hline
\end{tabular}

Table 1 The logic truth of the position protection

\section{The software design and implementation}

The system software design is mainly done in the DSP, including timer design, Hall pulse capture module, PWM generator module, A/D sampling module, serial communication module, the closed-loop control law design and the design for other data solver.

The timer module generates a timer interrupt whose period is 50us to gain various time bases. When the software runs, the system will call the appropriate modules just like the acquisition, the communication and the control unit and so on according to the various time bases. The PWM module generates a PWM wave whose amplitude is $3.3 \mathrm{~V}$, period is $20 \mathrm{us}$ and duty is adjustable. The execution period of the closed-loop control law is 50us and the output of the law is used to change the duty of the PWM. The capture module calculates to get the actual speed by capturing the width of the Hall pulse. 50us timing cycle will be set to gain the A/D conversion result for the closed-loop control. The serial communication is achieved by the serial interrupt. The module receives real-time data frames transmitted by the master device to get the control instructions, while the measured data will be packaged into frame data for sending to the master device every $10 \mathrm{~ms}$.

\section{Conclusions}

This paper presents a hardware design of digital servo system based on the control structure of DSP + CPLD. The integrated driver chip is adopted to make the realization of the system to be more simple and reliable. The system employs three closed loop control strategy including current loop, speed loop and position loop to achieve the fast and accurate response for the position command. Experiments show that the steady-state error is tininess and dynamic response is fast. The output rated load torque of the actuator is $100 \mathrm{Nm}$, so as to be suitable for the rudder servo control in the 
flight control of high-precision, large-load and high-speed.

\section{Acknowledgement}

In this paper, the research was sponsored by the National Nature Science Foundation under Grant 61104082.

\section{References}

[1] A. Boglietti, A. Cavagnino, A. Tenconi, and S. Vaschetto, "The safety critical electric machines and drives in the more electric aircraft: A survey," in 35th Annual Conference of IEEE Industrial Electronics (IECON 2009), Porto, Portugal, Nov. 2009, pp. 2587-2594.

[2] Chong Yang, Li Yanjun, Zhang ke, etal. Design and implementation of digital fuzzy-PID actuator control system[J].Flight Dynamincs, 2011, 29(2) : 86- 88. (in Chinese).

[3] Liu Tao, Lu Xunhong, Huang Yimin. The design of numeric rudder controller based on DSP[J]. Journal of Shenyang Institute of Aeronautical Engineering,2006, 23(1):8-11.(in Chinese)

[4] J. A. Rosero, J. A. Ortega, E. Aldabas, and L. Romeral, "Moving towards a more electric aircraft,” IEEE Aerosp. Electron. Syst. Mag., vol.22,no.3, pp.3-9,2007.

[5] Atkinson G J, Mecrow B C, Jack A G, et al. The Design of Fault Tolerant Machines for Aerospace Application [J]. IEEE International Conference on Electric Machines and Drives, 2005:1863-1869.

[6] A. Garcia, J. Cusido, J. A. Rosero, J. A. Ortega, and L. Romeral, "Reliable electro-mechanical actuators in aircraft,” IEEE Aerosp.Electron.Syst. Mag., vol. 23, no. 8, pp.19-25, 2008. 